\title{
Normal Tone-In-Noise Sensitivity in Trained Budgerigars despite Substantial Auditory-Nerve Injury: No Evidence of Hidden Hearing Loss
}

\author{
Kenneth S. Henry ${ }^{1,2,3}$ and Kristina S. Abrams ${ }^{3}$ \\ ${ }^{1}$ Department of Otolaryngology, University of Rochester, Rochester, New York 14642, ${ }^{2}$ Department of Biomedical Engineering, University of \\ Rochester, Rochester, New York 14642, and ${ }^{3}$ Department of Neuroscience, University of Rochester, Rochester, New York 14642
}

Loss of auditory-nerve (AN) afferent cochlear innervation is a prevalent human condition that does not affect audiometric thresholds and therefore remains largely undetectable with standard clinical tests. AN loss is widely expected to cause hearing difficulties in noise, known as "hidden hearing loss," but support for this hypothesis is controversial. Here, we used operant conditioning procedures to examine the perceptual impact of AN loss on behavioral tone-in-noise (TIN) sensitivity in the budgerigar (Melopsittacus undulatus; of either sex), an avian animal model with complex hearing abilities similar to humans. Bilateral kainic acid (KA) infusions depressed compound AN responses by $40-70 \%$ without impacting otoacoustic emissions or behavioral tone sensitivity in quiet. Surprisingly, animals with AN damage showed normal thresholds for tone detection in noise $(0.1 \pm 1.0 \mathrm{~dB}$ compared to control animals; mean difference $\pm \mathrm{SE})$, even under a challenging roving-level condition with random stimulus variation across trials. Furthermore, decision-variable correlations (DVCs) showed no difference for AN-damaged animals in their use of energy and envelope cues to perform the task. These results show that AN damage has less impact on TIN detection than generally expected, even under a difficult roving-level condition known to impact TIN detection in individuals with sensorineural hearing loss (SNHL). Perceptual deficits could emerge for different perceptual tasks or with greater AN loss but are potentially minor compared with those caused by SNHL.

Key words: cochlear synaptopathy; decision-variable correlation; hidden hearing loss; operant conditioning; sensorineural hearing loss

Significance Statement

Loss of auditory-nerve (AN) cochlear innervation is a common problem in humans that does not affect audiometric thresholds on a clinical hearing test. AN loss is widely expected to cause hearing problems in noise, known as "hidden hearing loss," but existing studies are controversial. Here, using an avian animal model with complex hearing abilities similar to humans, we examined for the first time the impact of an experimentally induced AN lesion on behavioral tone sensitivity in noise. Surprisingly, AN-lesioned animals showed no difference in hearing performance in noise or detection strategy compared with controls. These results show that perceptual deficits from AN damage are smaller than generally expected, and potentially minor compared with those caused by sensorineural hearing loss (SNHL).

Received Aug. 10, 2020; revised 0ct. 2, 2020; accepted 0ct. 24, 2021.

Author contributions: K.S.H. designed research; K.S.H. and K.S.A. performed research; K.S.H. and K.S.A. analyzed data; K.S.H. wrote the paper.

This work was supported by the National Institute on Deafness and Communication Disorders Grant R01DC017519 (to K.S.H). We thank Laurel Carney for providing equipment for behavioral tests and helpful comments on a previous draft of the manuscript; Wilson Geisler for providing MATLAB code for DVC analyses; Douglas Schwarz for assisting with software development; and Kassidy Amburgey, Nina Stash, and Margaret Youngman for assisting with behavioral experiments and analyses.

The authors declare no competing financial interests.

Correspondence should be addressed to Kenneth S. Henry at kenneth_henry@urmc.rochester.edu.

https://doi.org/10.1523/JNEUROSCI.2104-20.2020

Copyright $\odot 2021$ the authors

\section{Introduction}

Loss of auditory-nerve (AN) afferent innervation of the cochlea has long been recognized as a common pathology of aging (Otte et al., 1978). While traditionally considered as secondary to inner hair cell (IHC) death (Spoendlin, 1984; McFadden et al., 2004), recent studies show that age-related AN loss exceeds hair-cell loss by three to one ( $\mathrm{Wu}$ et al., 2019), and even occurs in individuals without overt haircell pathologies (Makary et al., 2011; Viana et al., 2015). Furthermore, up to $50 \%$ loss of AN synapses occurs in animal models following moderate noise exposures that are insufficient to produce permanent hair-cell damage (Kujawa and Liberman, 2009; Lin et al., 2011). These results suggest that the 
AN, rather than hair cells, may be the most vulnerable element of cochlear transduction.

AN loss in the absence of hair-cell damage does not elevate pure-tone thresholds in quiet and therefore remains undetectable with the audiogram (Makary et al., 2011; Schuknecht and Woellner, 1953), but has been proposed to cause deficits in realworld listening known as "hidden hearing loss" (Bharadwaj et al., 2014; Schaette and McAlpine, 2011). Evidence of hidden hearing loss in humans is mixed, with several studies showing associations between measures of AN health, prior noise exposure, and complex-sound perception (Liberman et al., 2016; Shehorn et al., 2020) but many others finding no relationships (Grose et al., 2017; Johannesen et al., 2019; Marmel et al., 2020; Le Prell et al., 2018; Prendergast et al., 2017, 2019; Yeend et al., 2017). These studies have typically estimated AN loss using Wave-I of the auditory brainstem response (ABR), an AN-generated potential with low signal-to-noise ratio (SNR) in humans, and focused on young, normal-hearing populations that may lack the variation in AN loss needed for an adequate test of hidden hearing loss. Because of these limitations, hidden hearing loss remains a topic of active debate and investigation.

Animal studies hold promise to identify perceptual changes with AN loss because neural lesions can be experimentally induced. However, only a small number of animal studies has assessed effects of AN loss on auditory perception, and none of these progressed beyond tone sensitivity in quiet. In cats, surgical AN damage does not elevate thresholds unless neural loss exceeds $80 \%$ (Schuknecht and Woellner, 1953). Similarly, most mice with $>95 \%$ AN loss because of ouabain exposure show normal tone sensitivity in quiet (Chambers et al., 2016). Finally, a recent study in budgerigars found no impact of 40$70 \%$ AN damage from kainic acid (KA), even for tone durations as short as $20 \mathrm{~ms}$ (Wong et al., 2019). Other studies based on acoustic-startle responses have found diverse changes in behavioral reflex strength that vary with the magnitude of AN loss and specific test paradigm (Hickox and Liberman, 2014; Chambers et al., 2016; Lobarinas et al., 2017), but translation of startle results to auditory perception remains controversial (Behrens and Klump, 2015, 2016).

The present study examined behavioral tone-in-noise (TIN) sensitivity in budgerigars with KA-induced AN damage. TIN sensitivity is thought to rely on robust encoding of energy and temporal envelope cues (Kidd et al., 1989; Richards, 1992; Kohlrausch et al., 1997; Mao et al., 2013, 2015; Mao and Carney, 2015) and, therefore, was expected to be adversely impacted by AN loss. Budgerigars were selected because this small parrot species shows behavioral performance similar to humans for many auditory tasks including frequency discrimination of tones and vowel formants (Dent et al., 2000; Henry et al., 2017a,b), amplitude modulation detection (Dooling and Searcy, 1981; Carney et al., 2013; Henry et al., 2016), and TIN detection (Dooling and Saunders, 1975; Saunders et al., 1978; Dooling et al., 2000). Moreover, a recent study showed that budgerigars use a weighted combination of energy and envelope cues, similar to that used by humans, when detecting low-frequency tones in noise (Henry et al., 2020). The present study used operant conditioning procedures to compare TIN sensitivity between budgerigars with and without AN damage.

\section{Materials and Methods}

Animals

Behavioral experiments were conducted in 12 adult budgerigars (Melopsittacus undulatus) of either sex under a protocol approved by the
University of Rochester Committee on Animal Resources. The experimental group $(n=5)$ was exposed bilaterally to KA as part of a previous study (Henry and Abrams, 2018) and consisted of three females and two males. The control group $(n=7)$ consisted of five females and two males. For animals in the KA group, the time interval between the final cochlear infusion and the onset of behavioral TIN testing ranged from 36 to 77 weeks. Behavioral sensitivity to tones in quiet was assessed after completion of TIN testing. A second control group of seven untrained animals was used for measurements of ABRs and distortion-product otoacoustic emissions (DPOAEs). Animals ranged in age from 1.5 to 5 years at the completion of testing.

\section{KA infusions}

AN damage was induced using bilateral cochlear infusions of KA, a glutamate analog that causes swelling and breakage of AN afferent synapses followed by irreversible loss of AN peripheral axons and cell bodies for sufficient exposures (Bledsoe et al., 1981; Pujol et al., 1985; Juiz et al., 1989; Sun et al., 2001). In birds, for which the length of the cochlear duct is relatively short (e.g., $\sim 2.5 \mathrm{~mm}$ in budgerigars; Manley et al., 1993), AN loss from KA is distributed diffusely across the full frequency range of hearing and associated with a concomitant reduction in the amplitude of compound AN responses (Sun et al., 2000; Henry and Abrams, 2018).

The methods used to induce AN damage with KA in budgerigars were described previously (Henry and Abrams, 2018; Wong et al., 2019). Briefly, anesthetized animals (3-5 mg/kg ketamine, $0.08-0.1 \mathrm{mg} / \mathrm{kg}$ dexmedetomidine, s.c.) were implanted with a head post and exposed bilaterally to KA during two cochlear infusion procedures, one for each ear. For each infusion, a craniotomy was opened by lifting a small flap of bone in the area rostral to the posterior semicircular canal and ventral to the horizontal canal, exposing the middle ear space and cochlear base (Konishi, 1964). A 150- $\mu$ m diameter cochleostomy was made into the scala vestibuli using gentle rotating hand pressure on a small drill bit. Thereafter, $2.5 \mu \mathrm{l}$ of 1 or $2 \mathrm{~mm} \mathrm{KA}$ (ab144490; Abcam) in hanks balanced salt solution (H8264; Sigma-Aldrich) was infused through the cochleostomy over $90 \mathrm{~s}$ using a microinjection syringe. Compound AN responses recorded from the cochleostomy site before and 5-10 min following KA infusions confirmed $>90 \%$ reduction of responses to clicks and 1- to 4$\mathrm{kHz}$ tones (Henry and Abrams, 2018). No attempt was made to close the cochleostomy given that a previous study found no changes in ABR Wave-I amplitude or thresholds one week after sham infusions of hanks balanced salt solution (Henry and Abrams, 2018; see also postinfusion DPOAE results from the present study). The bone flap was repositioned to close the craniotomy and the overlying skin incision closed with surgical adhesive.

\section{Free-field ABRs}

Free-field ABRs were recorded in experimental animals before KA infusions and at multiple time points thereafter, including after the completion of TIN behavioral testing, to assess longitudinal change in the amplitude of compound AN activity in response to clicks. Animals were anesthetized for ABR recordings as described above and transferred into an animal holder positioned on a table inside a sound attenuating booth. Body temperature was maintained from $39^{\circ} \mathrm{C}$ to $41^{\circ} \mathrm{C}$ using a heating pad (HTP-1500; Adroit Medical Systems). Stimuli were generated in MATLAB (R2015B; The MathWorks; 50-kHz sampling frequency) and converted to analog with 16-bit resolution by a data acquisition card (PCIe-6251; National Instruments). Level was calibrated based on the output of a $1 / 4$ " precision microphone (type 4938; Brüel \& Kjær) in response to tones, with the animal removed from the apparatus and the microphone placed at the location of the animal's head during ABR recordings. Stimulus level was controlled in two stages. First, up to $60 \mathrm{~dB}$ of analog attenuation was applied to the full-scale $( \pm 10 \mathrm{~V})$ input waveform by a computer-controlled attenuator (PA5; Tucker Davis Technologies). Second, when additional attenuation was required, the amplitude of the input waveform (generated by the NI card) was reduced to achieve the desired stimulus level. Note that this two-stage process maintained high stimulus amplitude resolution while restricting the range of analog attenuation to values for which attenuation error was $<0.1 \mathrm{~dB}$. Stimuli were amplified (D-75A, Crown Audio) and 
presented free field using a table-mounted loudspeaker (MC60; Polk Audio) positioned $20 \mathrm{~cm}$ from the animal and directed in the horizontal plane toward the dorsal surface of the head (the rostral surface faced downward in the animal holder).

Electrophysiological activity was recorded differentially between a vertex electrode [platinum subdermal needle (F-E2; Natus Manufacturing) or M0.6-threaded stainless steel rod implanted to the level of the dura] and two platinum needle electrodes inserted at the base of the skull near the nape (reference) and further down the back (ground). Activity was amplified by a factor of 50,000 and bandpass filtered from 30 to $10,000 \mathrm{~Hz}$ (P511; Grass Instruments) before sampling by the data acquisition card (50-kHz sampling frequency; 16-bit resolution) and storage on a computer hard drive. ABRs were recorded in response to 100- $\mu$ s monophasic clicks presented 19.2 times per second with alternating polarity in short sequences of increasing level from 30- to $80-\mathrm{dB}$ peak equivalent (p.e.) SPL. ABRs were calculated as the average response to 300 stimuli of each polarity (600 presentations total). Wave-I amplitude was calculated as the voltage difference between the first major positive peak of the ABR and the preceding baseline (Brittan-Powell et al., 2002).

\section{Closed-field ABRs and DPOAEs}

ABRs and DPOAEs were also recorded using a closed-field system at the time of TIN behavioral testing in all KA-exposed animals and a subgroup of the behaviorally trained controls. Additional recordings were made in a second control group of untrained animals $(n=7)$. Results were obtained in anesthetized animals using the same general procedures described above for free-field ABRs, except that a probe assembly consisting of a low-noise microphone (ER10-B+; Etymotic) and two earphones (Etymotic ER2) was sealed to each ear canal to produce a closed acoustic field. Stimulus amplification was provided by a headphone buffer (HB7; Tucker Davis Technologies) and the microphone signal was amplified by $40 \mathrm{~dB}$.

ABRs were evoked using click stimuli as described above, except that the left and right ears were stimulated diotically up to a maximum stimulus level of $90-\mathrm{dB}$ p.e. SPL. DPOAEs were recorded using the low-noise microphone in response to swept primary tones $F_{1}$ and $F_{2}$ with the frequency of $F_{1}$ increasing linearly from 0.5 to $6 \mathrm{kHz}$ over $4 \mathrm{~s}$ and $F_{2}$ frequency equal to $1.25 * \mathrm{~F}_{1}$. Primary tones were presented from separate earphones with equal sound level of 45 - to 70-dB SPL in 5-dB steps (six stimulus levels). Stimuli were $4.05 \mathrm{~s}$ in duration including $25-\mathrm{ms} \cos ^{2}$ onset and offset ramps and were presented in random sequence with a 300 -ms silent interval between successive stimuli. DPOAEs were calculated as the average microphone response to 20 stimulus presentations. DPOAE level at $2 * \mathrm{~F}_{1}-\mathrm{F}_{2}$ was extracted using a least squares fitting procedure (Long et al., 2008) in MATLAB using standard matrix operations, a 100-ms Hann window, and step size of $20 \mathrm{~ms}$. The same procedure was applied to a null response computed as the difference between the average even and odd-numbered stimulus presentations to estimate the noise floor. The average noise level at the distortion-product frequency was $0 \mathrm{~dB}$ at $\mathrm{F}_{2}$ of $800 \mathrm{~Hz}$ and less than $-10 \mathrm{~dB}$ at $\mathrm{F}_{2}$ values $>1 \mathrm{kHz}$.

\section{Behavioral TIN experiments}

Animals were trained to behaviorally detects tones in simultaneously gated noise using previously described materials and methods (Henry et al., 2020). Stimulus conditions included four test frequencies $(0.5,1,2$, and $4 \mathrm{kHz}$ ) and two noise levels (45- to 55- and 85-dB SPL), each of which was tested under fixed-level and roving-level conditions. Stimulus conditions were completed in different order across animals and tested repeatedly until behavioral sensitivity was stable, as described below. Noise was $1 / 3$ octave in bandwidth, log-centered on the tone frequency in all cases, and generated with a different random seed for each trial (seeds were recorded to allow subsequent analyses of trial-by-trial performance). Stimuli were $0.3 \mathrm{~s}$ in duration with $10-\mathrm{ms} \cos ^{2}$ onset and offset ramps.

Behavioral testing was performed in four foam-lined sound attenuating chambers (0.3- $\mathrm{m}^{3}$ internal volume; Industrial Acoustics). Animals perched under a loudspeaker (MC60; Polk Audio; driven by a Crown D$75 \mathrm{~A}$ amplifier) in the chamber with access to three response switches and the feeding tray of a seed dispenser (ENV-203; Med Associates; or 80209-20; Lafayette Instruments). The experimental apparatus was controlled by a computer containing a data acquisition card (PCI-6151; National Instruments) and running custom MATLAB software, a microcontroller (Arduino Leonardo), and custom hardware for conditioning input from the switches and output to the house light and seed dispenser. Stimuli were generated in MATLAB $(50-\mathrm{kHz}$ sampling frequency) and processed with a 5000-point digital preemphasis filter designed to correct for the frequency response of the system before ana$\log$ conversion by the data acquisition card. The preemphasis filter corrected both the magnitude and phase of presented stimuli, and was based on the output of a $1 / 2$ " precision microphone (type 4134 , Brüel \& Kjær) in response to $249 \log$-spaced tone frequencies from 0.05 to $15.1 \mathrm{kHz}$. The microphone was placed at the normal location of the animal's head during stimulus presentation, but with the animal removed from the apparatus. Overall level was controlled using the same twostage procedure described above for ABRs.

Animals started each trial by pecking the center switch, which initiated presentation of a single stimulus. The stimulus was either a "standard" noise-alone waveform or "target" tone-plus-noise waveform (50\% of trials each). The correct response to the standard noise stimulus was the right switch and the correct response to the target tone-plus-noise stimulus was the left switch. Correct and incorrect responses were reinforced by dispensing individual millet seeds and by "time outs" during which the light in the chamber was turned off, respectively. The number of dispensed seeds was adaptively varied throughout testing based on the most recent 50 trials to control response bias (Macmillan and Creelman, 2005). Test sessions with absolute bias $>0.3$ were excluded from further analysis. Behavioral testing was conducted 6-7 d per week in morning and afternoon blocks lasting $\sim 30$ min each.

Animals were initially trained to discriminate between the standard noise-alone stimulus and a high SNR (10-15 dB) target stimulus. Once $90 \%$ correct discrimination was reached, TIN sensitivity was assessed using two-down, one-up adaptive tracking procedures during which SNR varied within single test sessions (Levitt, 1971). The SNR of the target stimulus at the start of each tracking session was 10 to $15 \mathrm{~dB}$. Target SNR increased following each incorrect response to a target stimulus and decreased following two consecutive correct responses to target stimuli with the same SNR. Trials at which the direction of the "track" (SNR across target trials) changed from increasing to decreasing, or vice versa, were identified as reversals. The step size of the track decreased from an initial value of $3 \mathrm{~dB}$, to $2 \mathrm{~dB}$ after two reversals and to $1 \mathrm{~dB}$ after four reversals.

Within each track, the noise level remained fixed for a minimum of 15 reversals until two stability criteria were met: (1) the SD of the SNR of the final eight reversal points was $<3 \mathrm{~dB}$ and (2) the mean SNR difference between the final four reversal points and the preceding four reversals was $<3 \mathrm{~dB}$. Thereafter, the track continued under a "roving-level" condition for which the overall level of the stimulus was randomly scaled by $\pm 10 \mathrm{~dB}$ on each trial (uniform random distribution with $1-\mathrm{dB}$ resolution). The track continued for a minimum of 10 additional reversals until the same stability criteria defined above for the fixed-level condition were again satisfied. Animals typically completed four to six tracks per day consisting of 150-200 trials each. Reversal-based thresholds were calculated for fixed-level and roving-level portions of each track as the mean SNR of the final eight reversal points.

Tracking sessions were conducted repeatedly on the same condition until (1) at least 13 thresholds were obtained, (2) the SD of the final six track thresholds was $<3 \mathrm{~dB}$, and (3) the mean difference between the final three track thresholds and the preceding three was $<3 \mathrm{~dB}$. When all of these criteria were met for both fixed-level and roving-level thresholds, animals moved on to the next condition. Each animal completed the conditions in random sequence at least twice. Testing on a condition was discontinued when there was no significant threshold difference (two-sample $t$ test) from the previous testing block with the same stimuli. The total duration of behavioral testing ranged from four to five months across animals. Final thresholds were calculated for each condition and in each animal as the mean reversal-based threshold of the last 10 stable tracks. 


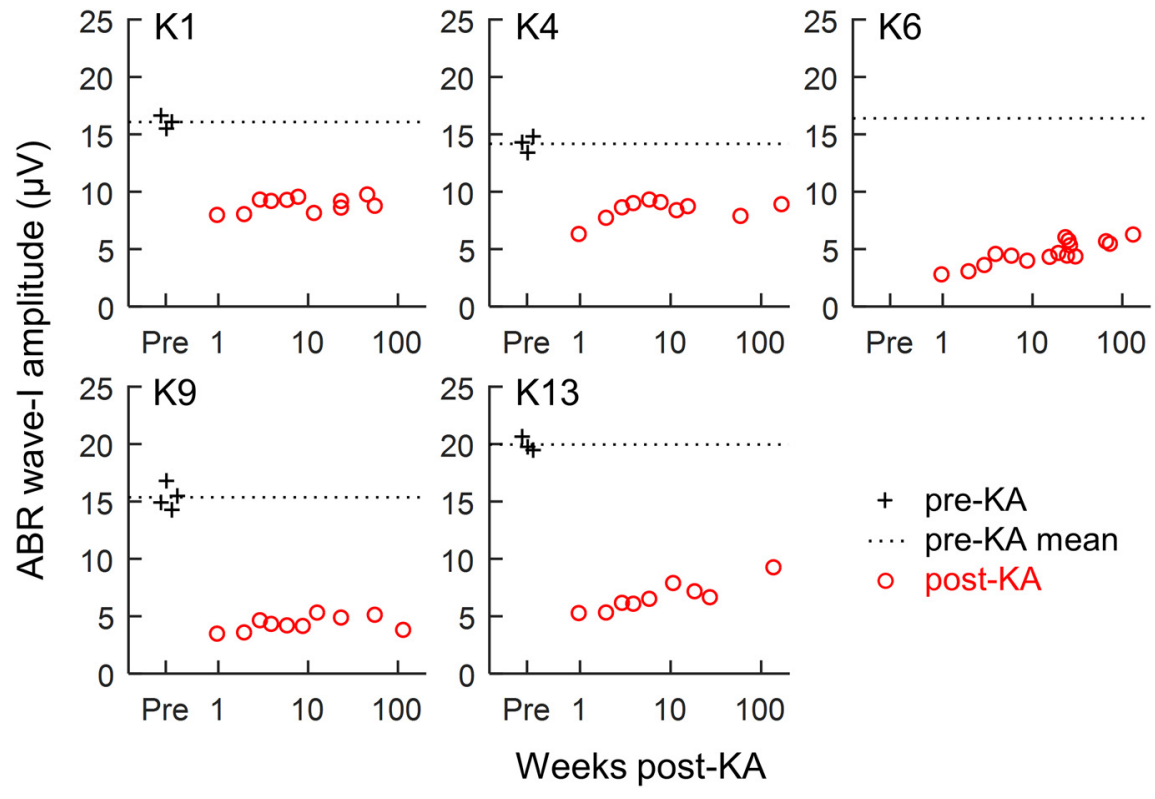

Figure 1. Change over time in ABR Wave-I amplitude following KA exposure. Animal identity is indicated in the top left of each panel. KA reduces Wave-I amplitude by $40-70 \%$ across animals, consistent with moderate-to-severe excitotoxic AN injury. Stimuli were free-field clicks presented at 80-dB p.e. SPL. Pre-KA amplitude was estimated for $K 6$ from measurements in the other animals because of a technical failure.

Behavioral sensitivity to tones in quiet

Behavioral sensitivity to tones in quiet was assessed using the same procedures and equipment described above for TIN testing, except that the target stimulus was a low-level, $2-\mathrm{kHz}$ tone, and no stimulus was presented on standard trials. Tones were $300 \mathrm{~ms}$ in duration with 10-ms $\cos ^{2}$ onset and offset ramps. Stimulus level was 40-dB SPL at the start of tracking sessions. The step size of the track decreased from an initial value of $10 \mathrm{~dB}$, to $5 \mathrm{~dB}$ after two reversals and to $2 \mathrm{~dB}$ after four reversals. Tracks continued for a minimum of 15 reversals until the SD of the final eight reversal points was $<4 \mathrm{~dB}$, and the mean difference between the last four reversals and the preceding four was $<3 \mathrm{~dB}$. Track thresholds were calculated as the mean of the final eight reversal points.

Tracking sessions were conducted repeatedly until (1) at least 13 reversal-based thresholds were obtained, (2) the SD of the final six track thresholds was $<4 \mathrm{~dB}$, and (3) the mean difference between the final three track thresholds and the preceding three was $<3 \mathrm{~dB}$. Animals began a new testing block when all of these criteria were met. Testing continued for at least two blocks until the absolute threshold difference compared with the previous testing block was $<2 \mathrm{~dB}$. Final thresholds were calculated in each animal as the mean reversal-based threshold of the last six stable tracks of the last and second to last testing blocks.

Decision-variable correlations (DVCs)

DVCs (Sebastian et al., 2017; Sebastian and Geisler, 2018) were used to evaluate the ability of a model combining energy and envelope cues to predict trial-by-trial behavioral responses of trained budgerigars to TIN stimuli. DVCs quantify the relationship between an observer's responses and those of a model tested with identical stimuli, where the model response is determined by an arbitrary decision variable. High DVCs provide evidence that the cues used to generate the model decision variable may be the ones used by animals as they perform the task. Procedures from Sebastian and Geisler (2018) were followed for maximum-likelihood estimation of DVCs in MATLAB. These methods provide separate estimates of the DVC for tone-plus-noise trials $\left(\rho_{T+N}\right)$ and noise trials $\left(\rho_{N}\right)$

The decision variable was calculated for each stimulus, shortened in duration to the animal's reaction time on that trial, as the weighted sum of single-channel energy and envelope cues. This relatively simple and physiologically grounded model explains greater variance in $500-\mathrm{Hz}$ TIN responses in this species than alternative models based on either cue or incorporating temporal fine structure information (Henry et al., 2020). The energy cue, $E$, was calculated as the root-meansquare amplitude of the stimulus waveform in decibels. The envelope cue, env, was calculated as the mean absolute first derivative of the Hilbert envelope of the stimulus waveform divided by the mean of the Hilbert envelope (Richards, 1992). Weights of each cue were calculated individually for each animal and stimulus condition as the coefficients of a multiple logistic regression analysis in MATLAB. The analysis included $E$ and env as independent variables and the observer's categorical response as the dependent variable. Standardized model coefficients $b *_{E}$ and $b_{e n v}^{*}$ are analogous to $R$ values produced by a correlation analysis (i.e., Pearson correlation coefficients) and, thus, can be used to compare the weighting of these cues in the observer's detection strategy. Standardization was accomplished by multiplying each raw coefficient by $\left(s_{X}\right)(R) / s_{\text {logit }(\hat{Y})}$, where $s_{X}$ is the $\mathrm{SD}$ of the predictor $X, R$ is the square root of the coefficient of determination of the full logistic regression model, and $s_{\text {logit }(\hat{Y})}$ is the SD of the logarithm of odds ratios predicted by the model (Menard, 2004). $b_{E}^{*}$ and $b^{*}{ }_{e n v}$ were computed separately for each trial type and averaged to produce final values, which was found based on model simulations to be necessary for unbiased estimation of the coefficients. The weighted energy-plus-envelope decision variable was calculated as $\left(b_{E}^{*}\right)(E) / s_{E}+\left(b^{*}{ }_{\text {env }}\right)(e n v) / s_{\text {env }}$.

\section{Statistical analyses}

Statistical analyses included two-sample $t$ tests conducted in MATLAB and linear mixed-effects models (Bates et al., 2015) conducted in R (version 3.6.2). Linear mixed-effects models included a random intercept for each subject to account for repeated measures and treated all independent variables (e.g., frequency, stimulus level, frequency range for the DPOAE analysis) as categorical. DPOAE level was defined as the median value of the response (thus minimizing the impact of notches when present) in half-octave $F_{2}$ ranges from 1 to $1.41,1.41$ to 2,2 to $2.83,2.83$ to 4 , and 4 to $5.66 \mathrm{kHz}$. Interactions were included in all analyses and dropped when not significant $(p>0.05)$ in order of decreasing $p$ value. Degrees of freedom for $F$ tests and pairwise comparisons of least-squared (LS) means were calculated based on the Satterthwaite approximation.

\section{Results}

\section{Bilateral KA exposure reduces compound AN activity without impacting otoacoustic emissions}

AN function was assessed in the five KA-exposed animals from the TIN behavioral experiments using ABR Wave-I, the compound response of the AN (Henry and Abrams, 2018). Free-field ABRs were recorded in response to click stimuli before and after bilateral KA exposure to quantify the extent of neural loss. ABRs recorded before KA exposure showed robust Wave-I amplitude that ranged from 15 to $20 \mu \mathrm{V}$ at $80-\mathrm{dB}$ p.e. SPL (Fig. 1). Wave-I amplitude decreased dramatically immediately following KA exposures before stabilizing at a fraction of the original preexposure value. Reduction of Wave-I amplitude extended throughout the period of behavioral testing and was statistically significant in all animals (Table 1). Based on responses recorded four or more weeks post-KA, the long-term average reduction of Wave-I ranged from $39.0 \%$ to $71.3 \%$ across animals, consistent with substantial AN injury. 
Table 1. ABR Wave-I reduction in KA-exposed budgerigars

\begin{tabular}{llllll}
\hline Animal & $\begin{array}{l}\text { Change in log Wave-I } \\
\text { amplitude } \\
\text { post-KA ( }\end{array}$ & $\begin{array}{l}\text { Percent Wave-I } \\
\text { reduction } \\
\text { post-KA }\end{array}$ & $t$ statistic & df & $p$ value \\
\hline K1 & $-0.586 \pm 0.037$ & $44.3 \%$ & -15.69 & 9 & $<0.0001$ \\
K4 & $-0.494 \pm 0.038$ & $39.0 \%$ & -12.88 & 8 & $<0.0001$ \\
K6 & -1.215 & $70.3 \%$ & & & \\
K9 & $-1.247 \pm 0.069$ & $71.3 \%$ & -17.96 & 9 & $<0.0001$ \\
K13 & $-1.036 \pm 0.09$. & $64.5 \%$ & -11.12 & 7 & $<0.0001$ \\
\hline
\end{tabular}

Two-sample $t$ tests. Wave-I reduction in $\mathrm{K} 6$ was estimated based on mean pre-KA amplitude of the other animals.

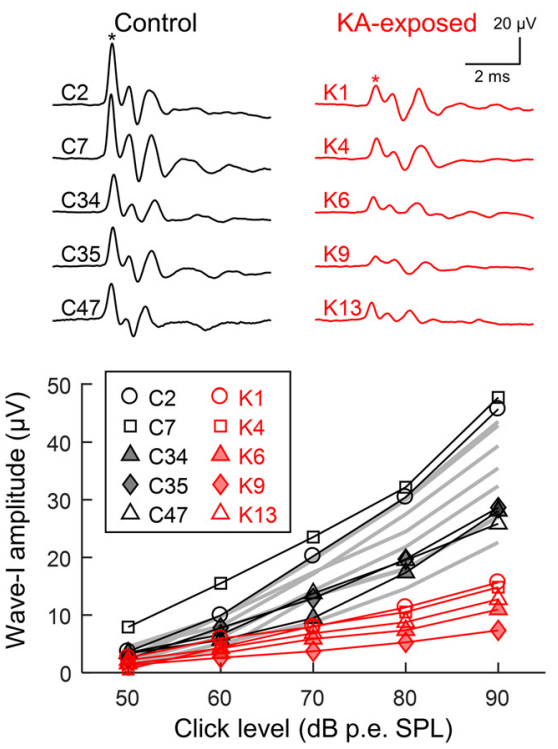

Figure 2. Comparison of closed-field ABRs between control and KA-exposed animals. ABR waveforms (top) are in response to closed-field clicks presented at 90-dB p.e. SPL. Stars indicate ABR Wave-I. Wave-I amplitude by click-level functions (bottom) in control and KAexposed animals from the TIN behavioral experiment compared with an untrained control group (gray lines; $n=7$ ). Wave-I reduction in KA-exposed animals is most pronounced at high stimulus levels.

ABRs were also recorded in response to closed-field clicks during the period of behavioral testing in the five KA-exposed animals, a subset of the behavioral control group (five of seven animals), and a second control group of untrained animals $(n=7$; Fig. 2). Wave-I amplitude in the behaviorally trained controls fell within the range observed in the untrained animals across a wide range of stimulus levels up to $90-\mathrm{dB}$ p.e. SPL, suggesting no difference in $\mathrm{AN}$ status between these groups. In contrast, KA-exposed animals showed considerably lower Wave-I amplitude. A linear mixed-effects model analysis of log-transformed Wave-I amplitude (to normalize residuals) showed significant effects of animal group (control vs KA exposed; $F_{(1,16)}=$ 29.06, $p<0.0001)$, stimulus level $\left(F_{(4,64)}=242.82, p<0.0001\right)$, and the animal group by level interaction $\left(F_{(4,64)}=2.60\right.$, $p=0.044)$. Compared with the control groups, mean Wave-I amplitude in KA-exposed animals was $47.1 \%$ lower at 50 -dB p.e. SPL $\left(-0.637 \pm 0.187 ; t_{(31.2)}=-3.41, p=0.0018\right.$; LS mean difference \pm SE in log-transformed Wave-I amplitude), $48.5 \%$ lower at $60-\mathrm{dB}$ p.e. SPL $\left(-0.663 \pm 0.187 ; t_{(31.2)}=-3.55, p=0.0012\right)$, $58.5 \%$ lower at $70-\mathrm{dB}$ p.e. SPL $\left(-0.879 \pm 0.187 ; t_{(31.2)}=-4.71\right.$, $p<0.0001), 63.2 \%$ lower at $80-\mathrm{dB}$ p.e. SPL $(-1.000 \pm 0.187$; $\left.t_{(31.2)}=-5.35, p<0.0001\right)$, and $64.4 \%$ lower at 90 -dB p.e. SPL $\left(-1.032 \pm 0.187 ; t_{(31.2)}=-5.53, p<0.0001\right)$. In contrast, the

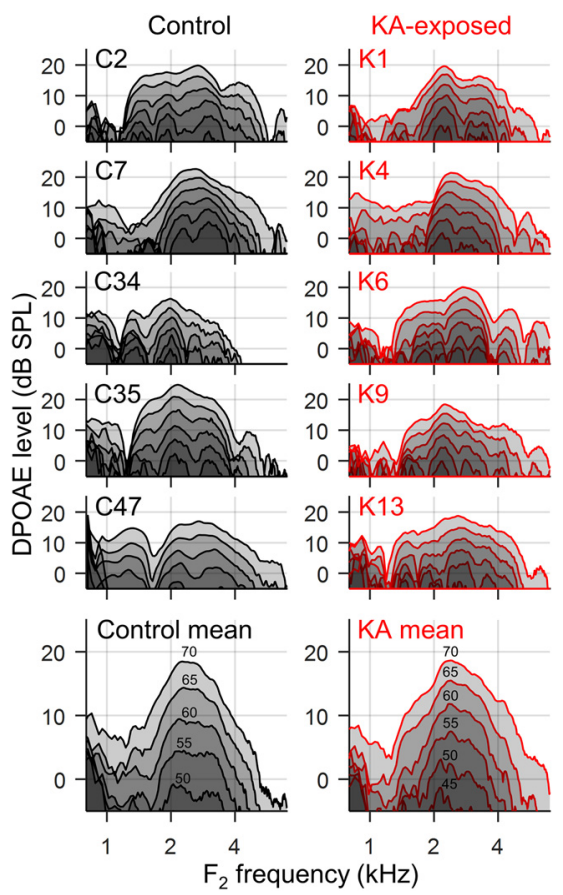

Figure 3. Comparison of DPOAE level between control (left) and KA-exposed animals (right). DPOAE level is shown at $2 F_{1}-F_{2}$ as a function of $F_{2}$ frequency. Stimuli were swept tone pairs with $F_{1}$ frequency increasing linearly from 0.5 to $6 \mathrm{kHz}$ over $4 \mathrm{~s}, F_{2}$ frequency of $1.25 * F_{1}$, and equal level of $F_{1}$ and $F_{2}$. Primary tone levels range from 45- to 70-dB SPL and are indicated in the bottom panels above the corresponding DPOAE curve. The average noise level at the distortion-product frequency was $0 \mathrm{~dB}$ at $F_{2}$ of $800 \mathrm{~Hz}$ and $<-10 \mathrm{~dB}$ at $F_{2}$ values $>1 \mathrm{kHz}$. DPOAE level is similar between KA-exposed and control groups from the TIN experiment despite substantial differences in Wave-I amplitude.

model confirmed no significant difference in Wave-I amplitude between the trained and untrained control groups $(-0.148 \pm$ $0.171 ; t_{(15)}=-0.86, p=0.40$; LS mean difference in log-transformed Wave-I amplitude \pm SE). These results show that at the time of behavioral testing, KA-exposed animals exhibited substantially lower compound AN activity than the behavioral control group and untrained controls.

DPOAEs were recorded to rule out the possibility that reduction of Wave-I amplitude in KA-exposed animals was because of inadvertent hair-cell trauma during the infusion procedures. DPOAEs were recorded in response to swept-tone stimuli in the same KA-exposed animals, trained controls, and untrained controls tested above (Fig. 3). DPOAE level increased with stimulus level and peaked at intermediate $\mathrm{F}_{2}$ frequencies from 2 to $3 \mathrm{kHz}$, within the frequency region of the budgerigar's best hearing sensitivity to tones (Dooling and Saunders, 1975). Little to no difference in DPOAE level was noted between KA-exposed and control animals. A linear mixed-effects model analysis of DPOAE level showed no main effect of $\mathrm{KA}\left(F_{(1,14)}=0.62\right.$, $p=0.44)$ but a significant interaction between KA exposure with $F_{2}$ range $\left(F_{(4,411.1)}=4.97, p=0.0006\right)$ because the difference between KA-exposed and control animals was slightly negative for the lowest $\mathrm{F}_{2}$ range $(1.56 \pm 1.59 \mathrm{~dB}$ for $1-1.41 \mathrm{kHz}$; LS mean difference $\pm \mathrm{SE}$ ) and slightly positive for higher $\mathrm{F}_{2}$ ranges (1.41$2 \mathrm{kHz}: 1.44 \pm 1.59 \mathrm{~dB} ; 2-2.83 \mathrm{kHz}: 2.48 \pm 1.59 \mathrm{~dB} ; 2.83-4 \mathrm{kHz}$ : $1.92 \pm 1.59 \mathrm{~dB} ; 4-5.66 \mathrm{kHz}: 1.48 \pm 1.59 \mathrm{~dB})$. Other significant effects in the model were $\mathrm{F}_{2}$ range $\left(F_{(4,411.1)}=249.87, p<\right.$ $0.0001)$, level $\left(F_{(5,411.3)}=416.65, p<0.0001\right)$, and the $F_{2}$ range by level interaction $\left(F_{(20,411.1)}=5.73, p<0.0001\right)$. These results suggest that substantial reduction of compound AN activity in KA- 


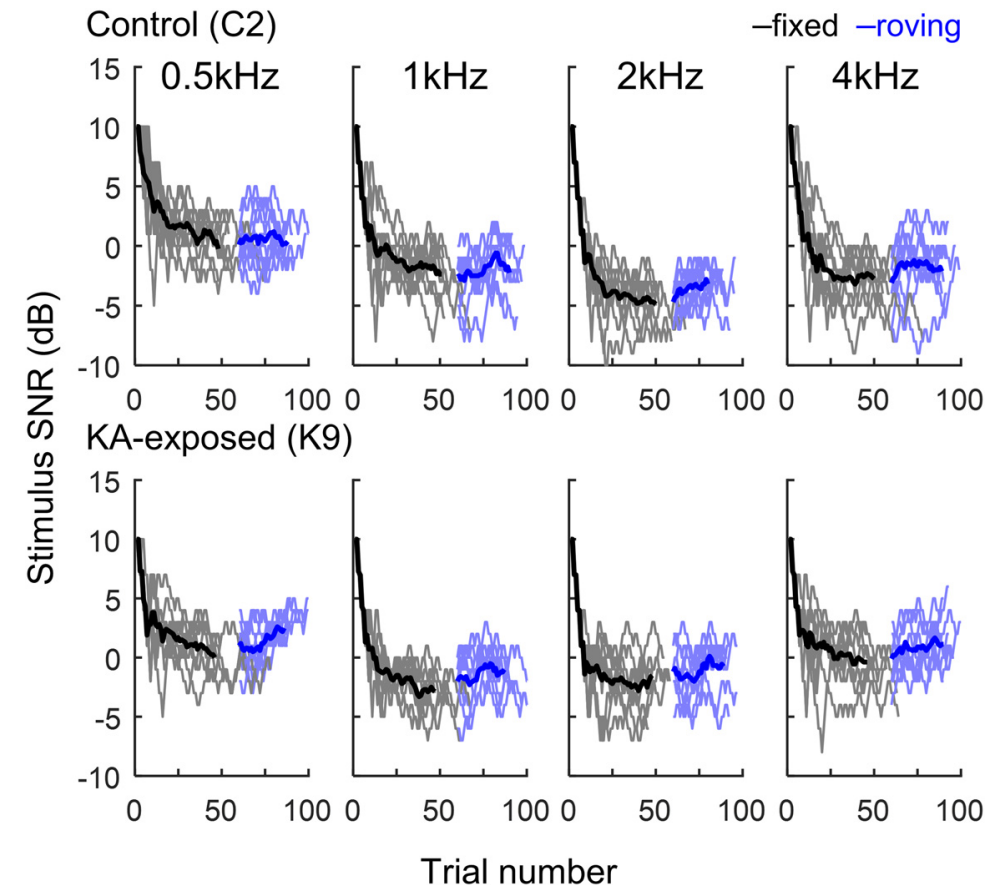

Figure 4. Representative two-down, one-up tracking behavioral tracking results. Traces show the SNR of TIN stimuli across test trials within individual sessions. Results are shown for one control animal (top) and one KA-exposed (bottom) under fixed-level and roving-level test conditions. Stimulus frequency is indicated at the top of each column. Noise level was $85-\mathrm{dB}$ SPL. Thick dark lines indicate mean SNR across 10 test sessions; thin lines show results from individual sessions. Behavioral performance is similar between animals and shows minimal change under the roving-level condition.

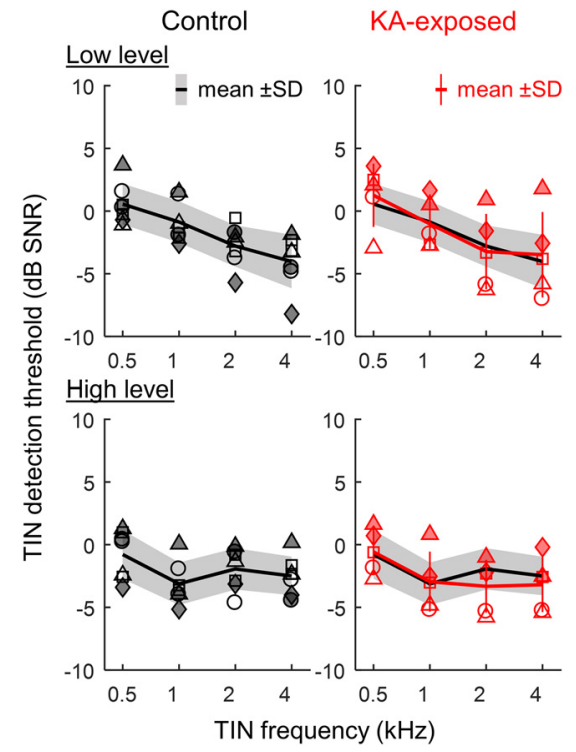

Figure 5. TIN detection thresholds of control (left) and KA-exposed animals (right) under the fixed-level condition. See Figure 2 for the animal identity of individual symbols in this and subsequent figures. Results are shown as a function of test frequency for low (top) and high (bottom) noise levels. TIN thresholds in KA-exposed animals are as sensitive as in the control group despite substantial reduction of ABR Wave-I.

exposed animals was because of neural/synaptic loss rather than a secondary consequence of hair-cell damage.

KA-exposed animals show normal behavioral sensitivity to TIN stimuli

Behavioral sensitivity to TIN stimuli was assessed in the five KAexposed animals and a group of seven controls to evaluate whether selective AN loss causes hidden perceptual deficits in noise. Testing was performed using a single-interval, twoalternative operant discrimination task, and two-down one-up adaptive tracking procedures (Levitt, 1971). Behavioral performance was assessed at four octavespaced test frequencies from 0.5 to $4 \mathrm{kHz}$ using simultaneously gated tone-plusnoise (target trials) and noise-alone stimuli (standard trials). Noise was one-third octave in bandwidth and log-centered on the tone frequency. Noise level was fixed during the first part of the test session at 55- or $85-\mathrm{dB}$ SPL for the $0.5-\mathrm{kHz}$ test frequency and at 45- or 85-dB SPL for higher test frequencies. Thereafter, the overall level of the stimulus varied over a $20-\mathrm{dB}$ range across trials (uniform random distribution; the roving-level condition), to test for a change in TIN sensitivity with decreased reliability of single-channel energy cues (Kidd et al., 1989; Richards, 1992; Leong et al., 2020).

Behavioral tracks showing target SNR across test trials decreased rapidly over 20-30 trials in all animals before stabilizing at the fixed-level TIN detection threshold (Fig. 4). Note that thresholds calculated from reversal points of the two-down one-up tracks correspond to $\sim 70.7 \%$ correct performance (Levitt, 1971). Across control animals, thresholds for the initial fixed-level portion of the track ranged from -5 to $+1 \mathrm{~dB}$ and decreased slightly with increasing test frequency, particularly for the low noise level (Fig. 5, left). Surprisingly, despite $40-70 \%$ persistent reduction of compound AN activity, thresholds of KA-exposed animals fell within the same range as those of the control group (Fig. 5, right). A linear mixed-effects model analysis found no significant impact of KA exposure on fixed-level TIN thresholds $\left(F_{(1,10)}=0.02, p=0.90\right)$ or interaction effect of KA exposure with frequency $\left(F_{(3,73)}=\right.$ $1.27, p=0.29)$ or stimulus level $\left(F_{(1,73)}=1.36, p=0.25\right)$. The estimated effect size of KA exposure on TIN thresholds was $-0.13 \pm 1.00 \mathrm{~dB}$ (mean $\pm \mathrm{SE}$; $95 \%$ confidence interval: $-2.37-$ $2.10 \mathrm{~dB}$ ), suggesting little or no impact (i.e., $<\sim 2 \mathrm{~dB}$ ) of AN loss on behavioral sensitivity to tones in fixed-level noise. Significant factors in the model included frequency $\left(F_{(3,73)}=35.49\right.$, $p<0.0001)$, stimulus level $\left(F_{(1,73)}=5.53, p=0.021\right)$, and the frequency by level interaction $\left(F_{(3,73)}=9.25, p<0.0001\right)$ because of different patterns of variation across test frequencies for low and high noise levels.

Random variation in stimulus level across trials (i.e., roving level) decreases the reliability of single-channel energy cues for TIN detection (Kidd et al., 1989; Richards, 1992) and thus is expected to cause threshold elevation proportional to the degree by which the subject uses single-channel energy cues to perform the task (Henry et al., 2020; Leong et al., 2020). In control animals, threshold shifts observed during the roving-level part of the testing session were generally slightly above $0 \mathrm{~dB}$ and almost invariably $<2 \mathrm{~dB}$ (Fig. 4, blue traces, Fig. 6, left), consistent with a small impact of the energy cue on task performance. Notably, KA-exposed animals showed similar roving-level threshold shifts to those observed in the control group (Fig. 6, right). A linear 


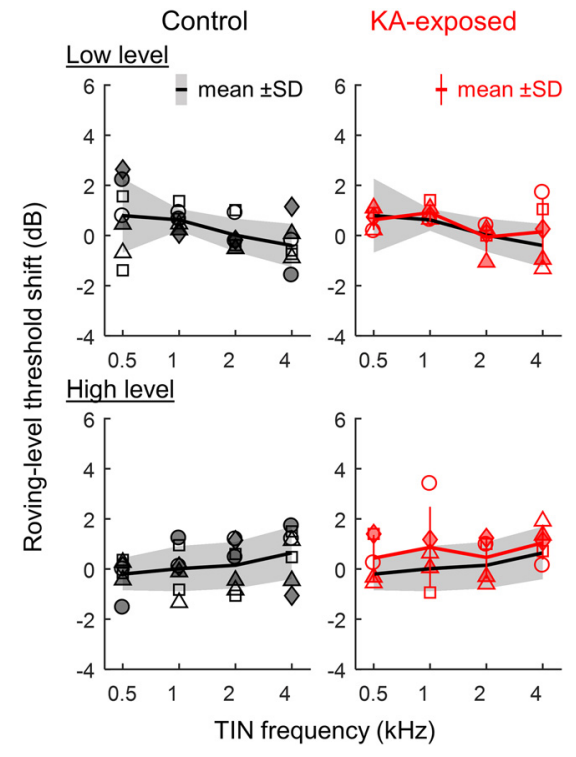

Figure 6. Threshold shifts in control (left) and KA-exposed animals (right) under the roving-level condition. Results are shown as a function of TIN frequency at low (top) and high (bottom) noise levels. Roving-level thresholds shifts are small in budgerigars and remarkably similar in magnitude between KA-exposed and normal-hearing animal groups.

mixed-effects model analysis of roving-level threshold shifts showed no main effect of KA exposure $\left(F_{(1,10)}=1.70, p=0.22\right)$ or interaction of KA exposure with frequency $\left(F_{(3,73)}=0.36\right.$, $p=0.78)$ or stimulus level $\left(F_{(1,73)}=1.31, p=0.26\right)$. The estimated impact of $\mathrm{KA}$ on the roving-level threshold shift was $0.34 \pm$ $0.27 \mathrm{~dB}$ (mean $\pm \mathrm{SE}$; 95\% confidence interval: $-0.24-0.95 \mathrm{~dB}$ ). The only significant model terms were the intercept $(0.71 \pm$ $\left.0.32 \mathrm{~dB} ; t_{(65.4)}=2.21, p=0.030\right)$, indicating the average rovinglevel threshold shift across conditions within an animal, and a frequency by stimulus level interaction $\left(F_{(3,73)}=4.52, p=0.0058\right)$ driven by a smaller rove effect with increasing frequency for the low stimulus level.

\section{KA-exposed animals show normal behavioral sensitivity to a $2-\mathrm{kHz}$ tone in quiet}

Sensitivity to a $2-\mathrm{kHz}$ tone in quiet was evaluated in all KAexposed animals and a subgroup of the controls $(n=5)$ at the conclusion of TIN behavioral experiments to test for possible differences in audiometric sensitivity. Thresholds measured from reversal points of two-down one-up adaptive tracks ranged from 6.2 - to $10.8-\mathrm{dB}$ SPL in the control group $(9.1 \pm 1.8-\mathrm{dB}$ SPL; mean $\pm \mathrm{SD}$ ), whereas thresholds in the KA-exposed group were 8.7-, 10.2-, 17.0-, 8.0-, and 10.2-dB SPL in animals K1, K4, K6, K9, and K13, respectively. These results suggest no significant impact of AN damage on audiometric sensitivity $\left(t_{(8)}=0.935\right.$, $p=0.38$; two-sample $t$ test), consistent with a previous report in these same KA-exposed animals that examined a wider range of tone frequencies $(0.25-8 \mathrm{kHz})$ and tone durations $(20-300 \mathrm{~ms}$; Wong et al., 2019).

\section{KA-exposed and control groups use the same energy-based and envelope-based cues for TIN detection}

DVCs quantify the ability of a model to predict trial-by-trial observer responses (Sebastian et al., 2017; Sebastian and Geisler, 2018), thereby providing greater potential insight into an individual's detection strategy than traditional analyses of detection thresholds alone. A previous study in normal-hearing budgerigars found that a decision variable combining single-channel energy and envelope cues could explain significantly greater variance in behavioral responses to $500-\mathrm{Hz}$ TIN stimuli than models based on either cue alone (Henry et al., 2020), a result consistent with numerous human studies of TIN detection implicating envelope-related detection strategies (Kidd et al., 1989; Richards, 1992; Kohlrausch et al., 1997; Davidson et al., 2006, 2009; Mao et al., 2013, 2015). Here, the same DVC approach and model were used to evaluate TIN detection strategies in budgerigars with normal hearing and KA-induced AN damage for frequencies from 0.5 to $4 \mathrm{kHz}$.

The decision variable of the energy-plus-envelope model was calculated for each stimulus as the weighted linear combination of single-channel energy and envelope cues. The energy cue $(E)$ was calculated as the root-mean-square amplitude of the stimulus in decibels. The envelope cue (env) was the mean absolute value of the first derivative of the stimulus envelope divided by the envelope mean (Richards, 1992), a metric that increases with the extent and frequency of envelope fluctuations in the stimulus and thus decreases with increasing TIN SNR (i.e., as the envelope flattens out because of the greater tone amplitude). Weights of $E$ and $e n v$ in the combined model decision variable were fit individually for each animal and stimulus condition using multiple logistic regression, where $E$ and $e n v$ were the independent variables, the dependent variable was the observer's categorical response (i.e., tone present or absent), and $b_{E}^{*}$ and $b^{*}{ }_{\text {env }}$ are used to denote standardized regression coefficients of the model. Analyses were restricted to trials from the roving-level portion of testing sessions $(\sim 500$ trials per condition in each animal $)$ to minimize collinearity of $E$ and $e n v$ cues.

Behavioral responses of control animals typically varied with the weighted $E+e n v$ decision variable, resulting in a DVC (Fig. 7 , right). The magnitude of the DVC was generally slightly greater for tone-plus-noise trials than noise trials, as observed previously (Henry et al., 2020), and somewhat lower for lowlevel, higher-frequency stimuli (Fig. 8, top left). Notably, DVCs in KA-exposed animals (Fig. 8, right; for representative results, see Fig. 7, lower half) were equal in magnitude to those of normal-hearing control animals, indicating no impact of AN damage on the extent to which the weighted $E+e n v$ decision variable could account for variability in trial-by-trial behavioral results. A linear mixed-effects model analysis found no effect of KA exposure on DVC $\left(F_{(1,10)}=0.98, p=0.34\right)$ or interactions of KA exposure with frequency $\left(F_{(3,167)}=0.64, p=0.59\right)$, stimulus level $\left(F_{(1,167)}=1.39, p=0.24\right)$, or trial type (tone-plus-noise vs noise; $\left.F_{(1,167)}=0.98, p=0.32\right)$. DVC was greater for tone-plusnoise trials than noise trials $(0.072 \pm 0.023$; LS mean difference $\left.\pm \mathrm{SE} ; F_{(1,167)}=10.29, p=0.0016\right)$ and showed stronger reduction with increasing frequency for the low stimulus level (level: $F_{(1,167)}=13.40, p=0.0003$; frequency: $F_{(3,167)}=5.87, p=0.0007$; frequency $\times$ level: $\left.F_{(3,167)}=10.94, p<0.0001\right)$.

Standardized logistic regression coefficients $b_{E}^{*}$ and $b_{\text {env }}^{*}$ are analogous to a Pearson's correlation coefficient, $R$, and hence provide information about the degree to which $E$ and env cues are weighted in each observer's TIN detection strategy. Most control animals showed positive values of $b_{E}^{*}$, a pattern associated with greater probability of reporting that the tone was present with increasing $E$ (Fig. 7, left). Across stimulus conditions, $b_{E}^{*}$ of control animals declined somewhat for low-level, higherfrequency stimuli (Fig. 9, left), with several animals at 2 and $4 \mathrm{kHz}$ actually showing negative values of $b_{E}^{*}$ (note that negative $b_{E}^{*}$ reduces TIN sensitivity because even under roving-level conditions, mean $E$ is slightly higher for tone-plus-noise trials than for noise trials; see $E$ distributions in Fig. 7). $b_{E}^{*}$ values in KA- 

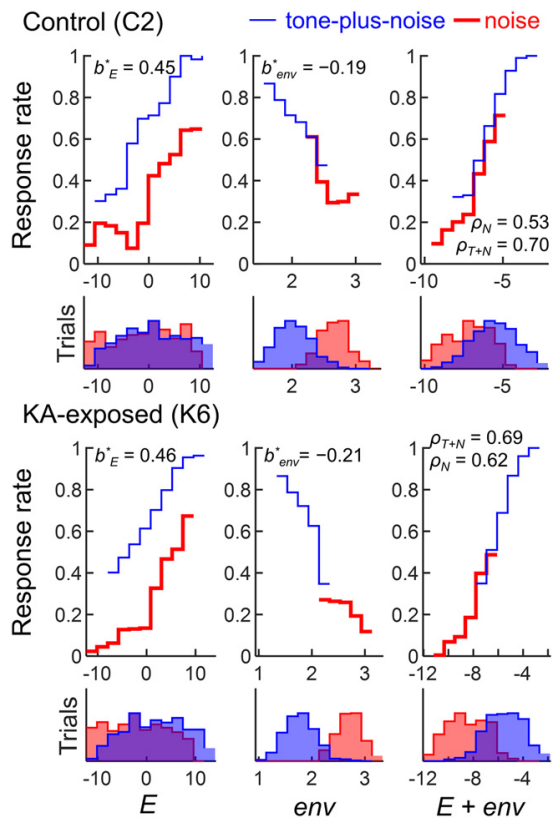

Figure 7. Variation of behavioral response rate with energy ( $E$; left), the envelope cue (env; middle), and the decision variable combining $E+$ env (right). Response rate is the proportion of trials for which the animal reported that the tone was present, with separate curves drawn for tone-plus-noise and noise trials. Results are shown from one control animal (top) and one KA-exposed (bottom) for the 1-kHz, high noise level condition. $b_{E}^{*}$ and $b^{*}{ }_{\text {env }}$ (left and middle panels) are standardized coefficients of a multiple logistic regression analysis and were used for cue weighting in the $E+$ env decision variable. DVCs (Sebastian et al., 2017; Sebastian and Geisler, 2018) are reported separately for each trial type (tone-plusnoise: $\rho_{T+N i}$ noise: $\rho_{N i}$ right panels) and indicate the extent to which $E+$ env accounts for trial-by-trial behavioral responses.

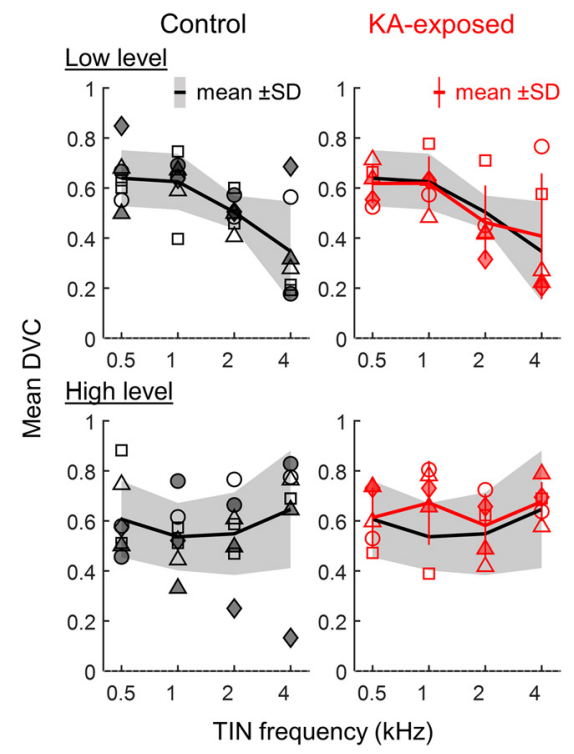

Figure 8. Mean DVCs of control (left) and KA-exposed animals (right) as a function of TIN frequency at low (top) and high (bottom) noise levels. DVCs are averaged across trial types, and indicate the extent to which $E+$ env accounts for behavioral responses to roving-level TIN stimuli. DVCs are highly similar between KA-exposed and normal-hearing animals.

exposed animals (Fig. 9, right) were similar in magnitude to those of normal hearing controls, indicating no difference in weighting of the single-channel energy cue for TIN detection. A linear mixed-effects model analysis found no effect of KA exposure on the magnitude of $b_{E}^{*}\left(F_{(1,10)}=1.19, p=0.30\right)$ or

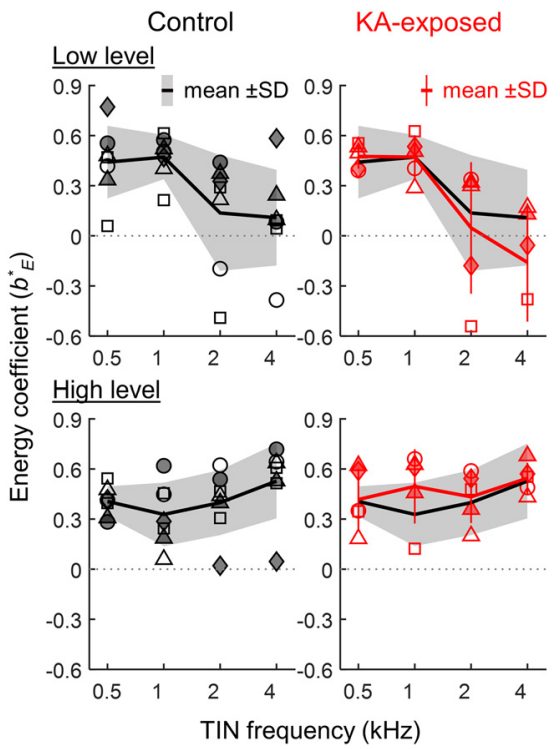

Figure 9. Standardized coefficients of the energy term $\left(b_{E}^{*}\right)$ in the $E+$ env model decision variable of control animals (left) and KA-exposed animals (right). Results are shown as a function of TIN frequency at low (top) and high (bottom) noise levels. $b^{*}{ }_{E}$ decreases for high-frequency, low-level stimuli, and is similar between KA-exposed and normal-hearing animals.

interactions of KA exposure with frequency $\left(F_{(3,73)}=0.18\right.$, $p=0.91)$ or stimulus level $\left(F_{(1,73)}=0.23, p=0.63\right)$. The interaction between frequency and level was significant $\left(F_{(3,73)}=5.84\right.$, $p=0.0012$ ) because of different patterns of variation across frequency for low and high stimulus levels.

Animals from both control and KA-exposed groups were invariably less likely to report that the tone was present with increasing env value (Fig. 7, center panels) and consequently showed negative values of $b^{*}{ }_{e n v}$ (Fig. 10). Note that negative $b_{\text {env }}^{*}$ benefits TIN sensitivity because env (i.e., the extent of envelope fluctuations) is lower for tone-plus-noise trials than for noise trials (see env distributions in Fig. 7, middle panels). $b_{e n v}^{*}$ was usually lower in magnitude than $b_{E}^{*}$ and remarkably similar between KA-exposed and normal-hearing animal groups (Fig. 10), suggesting no difference in the extent to which animals relied on envelope cues to perform the TIN detection task. A linear mixed-effects model analysis of $b^{*}{ }_{\text {env }}$ found no effect of KA exposure $\left(F_{(1,10)}=0.22, p=0.65\right)$ or interactions of KA exposure with frequency $\left(F_{(3,73)}=1.37, p=0.26\right)$ or level $\left(F_{(1,73)}=0.10\right.$, $p=0.76)$. The frequency by level interaction was significant $\left(F_{(3,73)}=4.94, p=0.0035\right)$ because of different patterns of variation across frequencies at high and low sound levels; $b_{\text {env }}^{*}$ increased slightly with increasing frequency for high-level stimuli (becoming less negative) and showed minimal variation with frequency for the lower level.

On average, the magnitude of $b^{*}$ env was roughly half that of $b_{E}^{*}$, raising the question why animals showed little of no threshold shift under the roving-level condition. Model simulations were used to explore the relationship between the weight of the envelope cue $\left(w_{\text {env }}\right)$ in the decision variable and the threshold shift under the roving-level condition. Simulations were conducted using fixed-level and roving-level $1000-\mathrm{Hz}$ TIN stimuli matched to the behavioral experiments, with 500 trials for each SNR. The decision variable of the model was calculated for values of $w_{\text {env }}$ ranging from 0 to 1 as $-w_{e n v} *$ env $+w_{E} * E$, where $w_{E}$ is the weighting of the energy term and $w_{E}$ and $w_{\text {env }}$ sum to 1 (i.e., extremes are the pure energy and envelope models). $E$ and env 


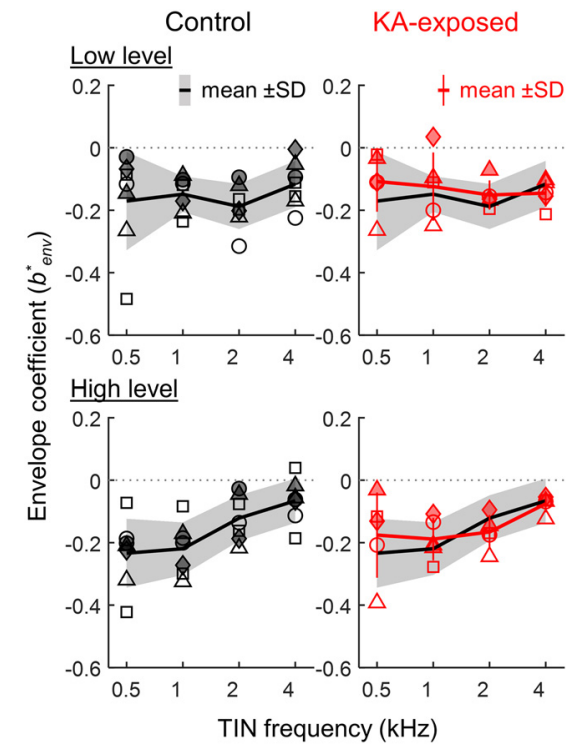

Figure 10. Standardized coefficients of the envelope term $\left(b^{*}{ }_{\text {env }}\right)$ in the $E+$ env model decision variable of control animals (left) and KA-exposed animals (right). Results are shown as a function of TIN frequency at low (top) and high (bottom) noise levels. $b^{*}{ }_{\text {env }}$ is invariably negative, indicating greater probability of responding that the tone is present when the normalized stimulus envelope shows less fluctuation and is similar between KA-exposed and normal-hearing animals.

were normalized before decision variable calculation by dividing by the SD observed for noise-alone trials. Thresholds were calculated using receiver operator characteristic analyses (Egan, 1975) as the minimum SNR above which separation between the decision-variable distribution for noise trials and the decision-variable distribution for tone-plus-noise trials exceeded $70.7 \%$.

Model thresholds under the fixed-level condition showed minimal variation across models, ranging from $-9.5 \mathrm{~dB}$ for the pure energy and envelope models to $-10.8 \mathrm{~dB}$ for intermediate models (Fig. 11, top row). Threshold shifts under the roving-level condition (Fig. 11, middle row and bottom panel) were greatest for the pure energy model as expected, at $12.6 \mathrm{~dB}$, and decreased with increasing $w_{e n v}$ to $4.7 \mathrm{~dB}$ for $w_{e n v}$ of 0.33 and $1.9 \mathrm{~dB}$ for $w_{e n v}$ of 0.67 . Note that for $w_{\text {env }}$ of 0.33 , which corresponds to the $\sim 2: 1$ ratio of $b_{E}^{*}$ to $b^{*}{ }_{\text {env }}$ observed in experimental animals, the model rove effect of $4.7 \mathrm{~dB}$ was larger than that observed in the TIN experiments $(0.71 \pm 0.32 \mathrm{~dB}$; mean difference $\pm \mathrm{SE}$; see above). It seems possible that animals incorporated a third cue in their decision process, which if independent of $E$ would further reduce the simulated rove effect below the value predicted by the basic $E+$ env model.

\section{Discussion}

In summary, budgerigars infused bilaterally with KA showed long-term 40-70\% reduction of compound AN activity without accompanying changes in DPOAE level or behavioral sensitivity to tones in quiet, a phenotype consistent with selective AN loss. While AN loss is generally thought to cause perceptual deficits in noise known as hidden hearing loss, KA-exposed animals instead showed normal behavioral thresholds for TIN detection across a wide range of conditions including a challenging roving-level task for which stimulus level varied randomly across trials. Furthermore, DVC analyses found no differences for KAexposed animals in the extent to which behavioral performance was related to energy and envelope cues.
This study is the first to our knowledge to evaluate the impact of an experimentally induced AN lesion on masked perception in trained animals. The estimated difference in TIN threshold between KA-exposed and control animals under the fixed-level condition was $-0.1 \pm 1.0 \mathrm{~dB}$ ( $\pm \mathrm{SE}$ ). While the finding that TIN sensitivity was not affected by AN loss may be unexpected, this result is consistent with model predictions based on signal detection theory showing thresholds shifts of only $1-3 \mathrm{~dB}$ for a variety of tasks over the physiological range of AN lesion sizes (40-70\%) shown by our KA-exposed animals (Oxenham, 2016). Indeed, based on this model framework, even a profound 90\% loss of AN afferents produces just $5 \mathrm{~dB}$ of threshold elevation for tone detection in both noisy and quiet backgrounds. Larger effects are predicted if the specific AN fibers removed by synaptopathy are of a more informative subtype than the fibers that survive (Furman et al., 2013; Carney, 2018), or if behavioral performance is limited primarily by central rather than peripheral sources of variability. In contrast, smaller effects are predicted when AN responses are more correlated across fibers (Oxenham, 2016).

A small impact of AN loss on perception might explain the mixed results produced by previous human studies, which have in most cases found no difference in hearing performance across normal-hearing listeners with different putative levels of AN loss (Grose et al., 2017; Prendergast et al., 2017, 2019; Yeend et al., 2017; Le Prell et al., 2018; Johannesen et al., 2019; Marmel et al., 2020). These studies focused on measures of masked speech or word recognition in young subjects, and inferred AN synaptopathy based on self-reported prior noise exposure - a potentially difficult approach considering that human subjects may vary in susceptibility to synaptic injury from noise and individuals under $\sim 50$ years of age rarely show $>20-30 \%$ loss of AN peripheral axons or cell bodies in postmortem studies (Makary et al., 2011; Viana et al., 2015; Wu et al., 2019). Despite these potential limitations, a few human studies have shown significant perceptual and physiological differences in individuals identified as at greater risk for AN synaptopathy, including lower recognition scores for masked and temporally altered speech, lower amplitude of ABR Wave-I when normalized by the summating potential, and reduced middle-ear reflex strength (Liberman et al., 2016; Shehorn et al., 2020).

Animal behavioral studies allow AN damage to be experimentally induced, an approach not possible in humans, but typically require weeks of training and behavioral testing to provide reliable estimates for auditory detection and discrimination performance for even a small number of conditions. While no previous animal study has assessed masked perception in $\mathrm{AN}$ lesioned animals to our knowledge, one study examined a potentially similar pathology involving selective loss of IHCs from carboplatin ototoxicity (Lobarinas et al., 2016). Carboplatinexposed chinchillas showed $40-80 \%$ IHC loss without a significant change in outer hair cell (OHC) survival or behavioral sensitivity to tones in quiet (Lobarinas et al., 2013, 2016). In contrast to the present results in budgerigars, TIN thresholds of chinchillas increased by $5-10 \mathrm{~dB}$ following IHC damage for both wideband noise and narrowband noise maskers centered on the tone frequency, similar to the stimuli employed here. Surprisingly, even greater impairment from IHC loss was observed for narrowband maskers with center frequencies different from the tone frequency, an unexpected result considering that $\mathrm{OHCs}$ and thus cochlear frequency tuning were thought to be normal in these animals. One possible explanation is that the perceptual deficits observed in carboplatin-treated animals were caused by physiological abnormalities of surviving IHCs, which can 

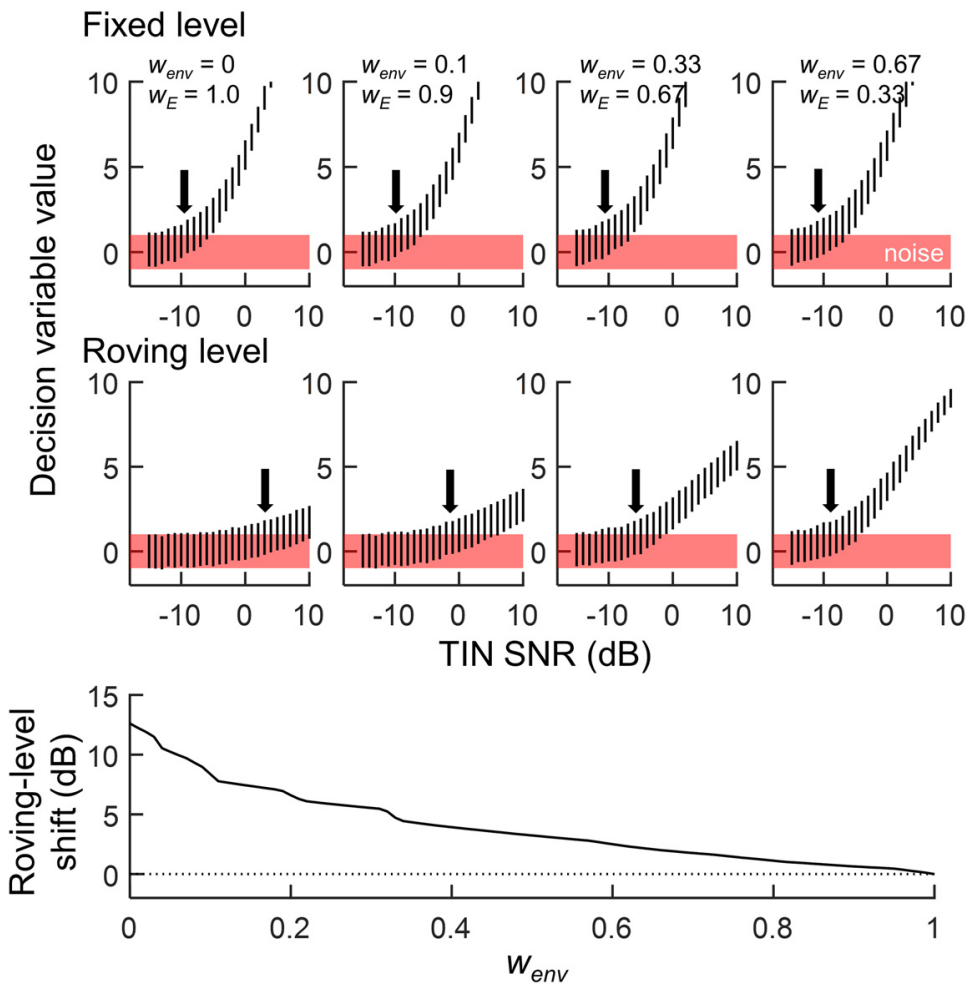

Figure 11. Simulations of fixed-level and roving-level TIN detection across different models. Model weights of the envelope cue $\left(w_{\text {env }}\right)$ and energy cue $\left(w_{E}\right)$ are indicated above the top row. Decision variable values (fixed level: top row; roving level: middle row) are normalized by the mean and the SD observed for noise trials and shown as function of SNR. Black vertical bars extend one SD above and below the mean decision-variable value for tone-plus-noise trials; red bands show the same for noise trials. Downward pointing arrows show the TIN threshold above which separation between the distributions of the two trial types exceeds $70.7 \%$. Model threshold shifts under the roving-level condition (bottom) are greatest for the pure energy model and increase with $w_{\text {env }}$.

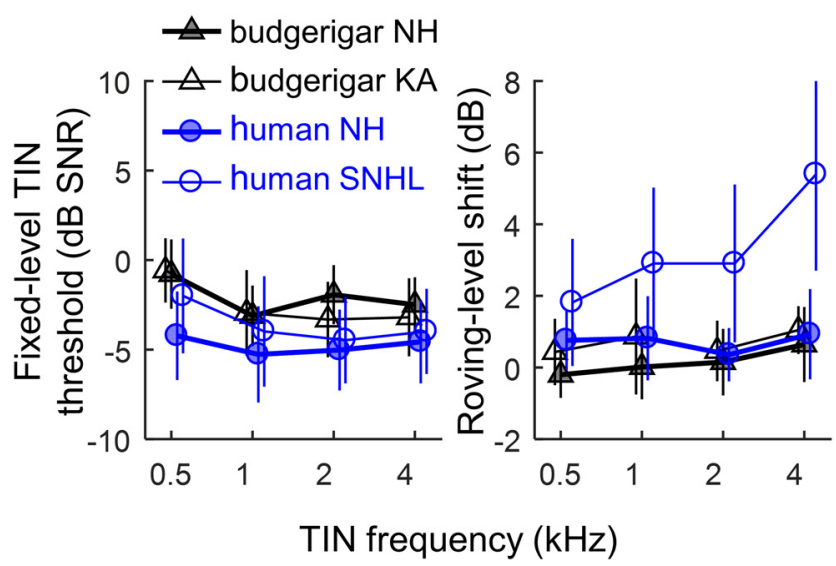

Figure 12. Comparison of TIN sensitivity between budgerigars and humans. Human results are from a previous study (Leong et al., 2020) with the same third-octave stimulus bandwidth and roving-level range as the present budgerigar study. Fixed-level TIN thresholds (left) are slightly more sensitive in humans than in budgerigars and minimally affected by SNHL. Normal-hearing budgerigars and humans show little or no threshold shift under the roving-level condition (right). SNHL in humans is associated with substantial roving-level threshold shifts, whereas AN damage in budgerigars is not.

become pronounced for large carboplatin doses associated with $>80 \%$ IHC death (Wang et al., 1997). A second possibility is that the degree of AN loss in chinchillas exceeded the $40-80 \%$ loss observed for IHCs, perhaps because of some "primary" AN loss for surviving IHCs in addition to the secondary loss attributable to IHC death. Consistent with this explanation, reduction of compound AN activity postcarboplatin has been shown to slightly exceed histologic IHC loss in several previous chinchilla studies (Trautwein et al., 1996; Wang et al., 1997).

Human subjects with mild-to-moderate sensorineural hearing loss (SNHL) were recently tested using the same 0.5to $4-\mathrm{kHz}$ TIN frequencies, third-octave stimulus bandwidth, and fixed-level and roving-level conditions of the present budgerigar study (Leong et al., 2020; Fig. 12). While diminished TIN sensitivity in individuals with SNHL might be anticipated based on the presence of both haircell pathology and AN synaptopathy, performance on the fixed-level condition was in fact normal from 1 to $4 \mathrm{kHz}$ and only slightly impaired $(\sim 2.5 \mathrm{~dB})$ for the 500 $\mathrm{Hz}$ test frequency (Fig. 12, left), a result likely attributable to the use of thirdoctave noise centered on the tone frequency (thus negating advantages of sharp cochlear frequency tuning). Notably, SNHL was associated with substantial threshold shifts under the roving-level condition, implicating greater reliance by listeners on single-channel energy cues (and less envelope-cue use) to performed the task (Fig. 12, right). Budgerigars approached the sensitivity of normal-hearing human subjects for the fixed-level condition and, in contrast to human subjects with SNHL, showed little or no impairment under the roving-level condition, even when AN loss from KA was substantial. These results suggest that unlike SNHL in humans, AN damage may have little impact on envelope-cue use for TIN detection.

Additional evidence that $\mathrm{AN}$ damage does not impact the cues used for TIN detection comes from the DVC analyses, which showed that trial-by-trial behavioral responses were predictable by a simple model combining energy and envelope cues in all animals regardless of AN status. TIN detection patterns of normal-hearing human subjects are also best explained by models combining energy and envelope cues (Richards, 1992; Kohlrausch et al., 1997; Mao et al., 2013), and notably, show stronger weighting of energy with SNHL that increases with the severity of hearing loss (Mao et al., 2015). Together with the roving-level threshold shifts discussed above, these results raise the possibility that perceptual deficits from AN loss may be minor compared with those associated with SNHL, even for tasks not reliant on sharp cochlear frequency tuning such as tone detection in narrowband noise.

The avian cochlea is shorter than in mammals, uncoiled, and wider with up to 30 hair cells across its width. Hair-cell shape changes gradually across the width of the sensory epithelium from "tall" to "short," with tall hair cells receiving the bulk of AN afferent innervation with up to four synapses each (Takasaka and Smith, 1971; Gleich, 1989; Smolders et al., 1995; Köppl et al., 2000). Thus, afferent synaptic density is $\sim 75 \%$ lower than in mammalian IHCs, raising the possibility that some epithelial regions might become completely deafferented following KA in 
birds. Further study is needed to investigate this hypothesis, including possible perceptual consequences. AN response properties are unknown in budgerigars, but in other birds include irregular spontaneous activity and V-shaped tuning curves similar to those found in cat (Sachs et al., 1974; Manley et al., 1985; Salvi et al., 1992). Other similar response properties include the dynamic range of rate-level functions and phase-locking limits (Gleich and Klump, 1995). In contrast, spontaneous activity is unimodally distributed in birds rather than bimodal as in mammals, and units without spontaneous activity are not observed. In light of these basic physiological similarities, as well as findings of human-like behavioral performance in normal-hearing budgerigars (Saunders et al., 1979; Henry et al., 2017b, 2020; Dent et al., 2000; Dooling et al., 2000), it seems promising that behavioral results in AN-damaged budgerigars might be observed in humans or mammalian animal models for the same degree of neural loss.

In conclusion, budgerigars showed normal behavioral sensitivity to TIN stimuli despite $40-70 \%$ reduction of compound AN activity because of KA, even for a difficult roving-level condition with random variation in stimulus level across trials. DVC analyses of trial-by-trail behavioral results showed no clear difference in KA-exposed animals compared with controls in the balance of energy and envelope cues used to perform the task. These results contrast with previous findings in humans with SNHL, who show perceptual impairment under the roving-level condition and increased correlation of behavioral responses to energy cues (Mao et al., 2015; Leong et al., 2020). Perceptual deficits from AN loss may be smaller than expected for $40-70 \%$ loss of fibers and may be minor compared with those caused by SNHL.

\section{References}

Bates D, Mächler M, Bolker B, Walker S (2015) Fitting linear mixed-effects models using lme4. J Stat Softw 67:1-48.

Behrens D, Klump GM (2015) Comparison of the sensitivity of prepulse inhibition of the startle reflex and operant conditioning in an auditory intensity difference limen paradigm. Hear Res 321:35-44.

Behrens D, Klump GM (2016) Comparison of mouse minimum audible angle determined in prepulse inhibition and operant conditioning procedures. Hear Res 333:167-178.

Bharadwaj HM, Verhulst S, Shaheen L, Liberman MC, Shinn-Cunningham BG (2014) Cochlear neuropathy and the coding of supra-threshold sound. Front Syst Neurosci 8:26.

Bledsoe SC, Bobbin RP, Chihal DM (1981) Kainic acid: an evaluation of its action on cochlear potentials. Hear Res 4:109-120.

Brittan-Powell EF, Dooling RJ, Gleich O (2002) Auditory brainstem responses in adult budgerigars (Melopsittacus undulatus). J Acoust Soc Am 112:999-1008.

Carney LH (2018) Supra-threshold hearing and fluctuation profiles: implications for sensorineural and hidden hearing loss. J Assoc Res Otolaryngol 19:331-352.

Carney LH, Ketterer AD, Abrams KS, Schwarz DM, Idrobo F (2013) Detection thresholds for amplitude modulations of tones in budgerigar, rabbit, and human. Adv Exp Med Biol 787:391-398.

Chambers AR, Resnik J, Yuan Y, Whitton JP, Edge AS, Liberman MC, Polley DB (2016) Central gain restores auditory processing following near-complete cochlear denervation. Neuron 89:867-879.

Davidson SA, Gilkey RH, Colburn HS, Carney LH (2006) Binaural detection with narrowband and wideband reproducible noise maskers. III. Monaural and diotic detection and model results. J Acoust Soc Am 119:2258-2275.

Davidson SA, Gilkey RH, Colburn HS, Carney LH (2009) An evaluation of models for diotic and dichotic detection in reproducible noises. J Acoust Soc Am 126:1906-1925.

Dent ML, Dooling RJ, Pierce AS (2000) Frequency discrimination in budgerigars (Melopsittacus undulatus): effects of tone duration and tonal context. J Acoust Soc Am 107:2657-2664.
Dooling RJ, Saunders JC (1975) Hearing in the parakeet (Melopsittacus undulatus): absolute thresholds, critical ratios, frequency difference limens, and vocalizations. J Comp Physiol Psychol 88:1-20.

Dooling RJ, Searcy MH (1981) Amplitude modulation thresholds for the parakeet (Melopsittacus undulatus). J Comp Physiol 143:383-388.

Dooling RJ, Lohr B, Dent ML (2000) Hearing in birds and reptiles. In: Comparative hearing: birds and reptiles (Dooling RJ, Fay RR, Popper AN, eds), pp 308-359. New York: Springer.

Egan JP (1975) Signal detection theory and ROC analysis (Carterette EC and Friedman MP, eds). New York: Academic Press.

Furman AC, Kujawa SG, Liberman MC (2013) Noise-induced cochlear neuropathy is selective for fibers with low spontaneous rates. J Neurophysiol 110:577-586.

Gleich O (1989) Auditory primary afferents in the starling: correlation of function and morphology. Hear Res 37:255-267.

Gleich O, Klump GM (1995) Temporal modulation transfer functions in the European starling (Sturnus vulgaris): II. Responses of auditory-nerve fibres. Hear Res 82:81-92.

Grose JH, Buss E, Hall JW (2017) Loud music exposure and cochlear synaptopathy in young adults: isolated auditory brainstem response effects but no perceptual consequences. Trends Hear 21:233121651773741.

Henry KS, Abrams KS (2018) Persistent auditory nerve damage following kainic acid excitotoxicity in the budgerigar (Melopsittacus undulatus). J Assoc Res Otolaryngol 19:435-449.

Henry KS, Neilans EG, Abrams KS, Idrobo F, Carney LH (2016) Neural correlates of behavioral amplitude modulation sensitivity in the budgerigar midbrain. J. Neurophysiol 115:1905-1916.

Henry KS, Abrams KS, Forst J, Mender MJ, Neilans EG, Idrobo F, Carney LH (2017a) Midbrain synchrony to envelope structure supports behavioral sensitivity to single-formant vowel-like sounds in noise. J Assoc Res Otolaryngol 18:165-181.

Henry KS, Amburgey KN, Abrams KS, Idrobo F, Carney LH (2017b) Formant-frequency discrimination of synthesized vowels in budgerigars (Melopsittacus undulatus) and humans. J Acoust Soc Am 142:2073-2083.

Henry KS, Amburgey KN, Abrams KS, Carney LH (2020) Identifying cues for tone-in-noise detection using decision variable correlation in the budgerigar (Melopsittacus undulatus). J Acoust Soc Am 147:984-997.

Hickox AE, Liberman MC (2014) Is noise-induced cochlear neuropathy key to the generation of hyperacusis or tinnitus? J Neurophysiol 111:552564 .

Johannesen PT, Buzo BC, Lopez-Poveda EA (2019) Evidence for age-related cochlear synaptopathy in humans unconnected to speech-in-noise intelligibility deficits. Hear Res 374:35-48.

Juiz JM, Rueda J, Merchán JA, Sala ML (1989) The effects of kainic acid on the cochlear ganglion of the rat. Hear Res 40:65-74.

Kidd G, Mason CR, Brantley MA, Owen GA (1989) Roving-level tone-innoise detection. J Acoust Soc Am 86:1310-1317.

Kohlrausch A, Fassel R, Van Der Heijden M, Kortekaas R, Van De Par S, Oxenham AJ, Püschel D (1997) Detection of tones in low-noise noise: further evidence for the role of envelope fluctuations. Acustica 83:659669.

Konishi M (1964) Effects of deafening on song development in two species of juncos. Condor 66:85-102.

Köppl C, Wegscheider A, Gleich O, Manley GA (2000) A quantitative study of cochlear afferent axons in birds. Hear Res 139:123-143.

Kujawa SG, Liberman MC (2009) Adding insult to injury: cochlear nerve degeneration after "temporary" noise-induced hearing loss. J Neurosci 29:14077-14085.

Leong UC, Schwarz DM, Henry KS, Carney LH (2020) Sensorineural hearing loss diminishes use of temporal envelope cues: evidence from roving-level tone-in-noise detection. Ear Hear 41:1009-1019.

Le Prell CG, Siburt HW, Lobarinas E, Griffiths SK, Spankovich C (2018) No reliable association between recreational noise exposure and threshold sensitivity, distortion product otoacoustic emission amplitude, or wordin-noise performance in a college student population. Ear Hear 39:10571074.

Levitt H (1971) Transformed up-down methods in psychoacoustics. J Acoust Soc Am 49:467-477.

Liberman MC, Epstein MJ, Cleveland SS, Wang H, Maison SF (2016) Toward a differential diagnosis of hidden hearing loss in humans. PLoS One 11:e0162726. 
Lin HW, Furman AC, Kujawa SG, Liberman MC (2011) Primary neural degeneration in the guinea pig cochlea after reversible noise-induced threshold shift. J Assoc Res Otolaryngol 12:605-616.

Lobarinas E, Salvi R, Ding D (2013) Insensitivity of the audiogram to carboplatin induced inner hair cell loss in chinchillas. Hear Res 302:113-120.

Lobarinas E, Salvi R, Ding D (2016) Selective inner hair cell dysfunction in chinchillas impairs hearing-in-noise in the absence of outer hair cell loss. J Assoc Res Otolaryngol 17:89-101.

Lobarinas E, Spankovich C, Le Prell CG (2017) Evidence of "hidden hearing loss" following noise exposures that produce robust TTS and ABR wave-I amplitude reductions. Hear Res 349:155-163.

Long GR, Talmadge CL, Lee J (2008) Measuring distortion product otoacoustic emissions using continuously sweeping primaries. J Acoust Soc Am 124:1613-1626.

Macmillan NA, Creelman CD (2005) Detection theory: a user's guide, Ed 2. Mahwah: Lawrence Erlbaum Associates Publishers.

Makary CA, Shin J, Kujawa SG, Liberman MC, Merchant SN (2011) Agerelated primary cochlear neuronal degeneration in human temporal bones. J Assoc Res Otolaryngol 12:711-717.

Manley GA, Gleich O, Leppelsack HJ, Oeckinghaus H (1985) Activity patterns of cochlear ganglion neurones in the starling. J Comp Physiol A Neuroethol Sens Neural Behav Physiol 157:161-181.

Manley GA, Schwabedissen G, Gleich O (1993) Morphology of the basilar papilla of the budgerigar, Melopsittacus undulatus. J Morphol 218:153165

Mao J, Carney LH (2015) Tone-in-noise detection using envelope cues: comparison of signal-processing-based and physiological models. J Assoc Res Otolaryngol 16:121-133.

Mao J, Vosoughi A, Carney LH (2013) Predictions of diotic tone-in-noise detection based on a nonlinear optimal combination of energy, envelope, and fine-structure cues. J Acoust Soc Am 134:396-406.

Mao J, Koch KJ, Doherty KA, Carney LH (2015) Cues for diotic and dichotic detection of a $500-\mathrm{Hz}$ tone in noise vary with hearing loss. J Assoc Res Otolaryngol 16:507-521.

Marmel F, Cortese D, Kluk K (2020) The ongoing search for cochlear synaptopathy in humans: masked thresholds for brief tones in threshold equalizing noise. Hear Res 392:107960.

McFadden SL, Ding DL, Jiang H, Salvi R (2004) Time course of efferent fiber and spiral ganglion cell degeneration following complete hair cell loss in the chinchilla. Brain Res 997:40-51.

Menard S (2004) Six approaches to calculating standardized logistic regression coefficients. Am Stat 58:218-223.

Otte J, Schunknecht HF, Kerr AG (1978) Ganglion cell populations in normal and pathological human cochleae. Implications for cochlear implantation. Laryngoscope 88:1231-1246.

Oxenham AJ (2016) Predicting the perceptual consequences of hidden hearing loss. Trends Hear 20:233121651668676.

Prendergast G, Millman RE, Guest H, Munro KJ, Kluk K, Dewey RS, Hall DA, Heinz MG, Plack CJ (2017) Effects of noise exposure on young adults with normal audiograms II: behavioral measures. Hear Res 356:74-86.

Prendergast G, Couth S, Millman RE, Guest H, Kluk K, Munro KJ, Plack CJ (2019) Effects of age and noise exposure on proxy measures of cochlear synaptopathy. Trends Hear 23:2331216519877301.

Pujol R, Lenoir M, Robertson D, Eybalin M, Johnstone BM (1985) Kainic acid selectively alters auditory dendrites connected with cochlear inner hair cells. Hear Res 18:145-151.
Richards VM (1992) The detectability of a tone added to narrow bands of equal-energy noise. J Acoust Soc Am 91:3424-3435.

Sachs MB, Young ED, Lewis RH (1974) Discharge patterns of single fibers in the pigeon auditory nerve. Brain Res 70:431-447.

Salvi R, Saunders SS, Powers NL, Boettcher FA (1992) Discharge patterns of cochlear ganglion neurons in the chicken. J Comp Physiol A Neuroethol Sens Neural Behav Physiol 170:227-241.

Saunders JC, Denny RM, Bock GR (1978) Critical bands in the parakeet (Melopsittacus undulatus). J Comp Physiol 125:359-365.

Saunders JC, Rintelmann WF, Bock GR (1979) Frequency selectivity in bird and man: a comparison among critical ratios, critical bands and psychophysical tuning curves. Hear Res 1:303-323.

Schaette R, McAlpine D (2011) Tinnitus with a normal audiogram: physiological evidence for hidden hearing loss and computational model. J Neurosci 31:13452-13457.

Schuknecht HF, Woellner RC (1953) Hearing losses following partial section of the cochlear nerve. Laryngoscope 63:441-465.

Sebastian S, Geisler WS (2018) Decision-variable correlation. J Vis 18:1-19.

Sebastian S, Abrams J, Geisler WS (2017) Constrained sampling experiments reveal principles of detection in natural scenes. Proc Natl Acad Sci USA 114:E5731-E5740.

Shehorn J, Strelcyk O, Zahorik P (2020) Associations between speech recognition at high levels, the middle ear muscle reflex and noise exposure in individuals with normal audiograms. Hear Res 392:107982.

Smolders JW, Ding-Pfennigdorff D, Klinke R (1995) A functional map of the pigeon basilar papilla: correlation of the properties of single auditory nerve fibres and their peripheral origin. Hear Res 92:151-169.

Spoendlin H (1984) Factors inducing retrograde degeneration of the cochlear nerve. Ann Otol Rhinol Laryngol Suppl 112:76-82.

Sun H, Salvi R, Ding DL, Hashino DE, Shero M, Zheng XY (2000) Excitotoxic effect of kainic acid on chicken otoacoustic emissions and cochlear potentials. J Acoust Soc Am 107:2136-2142.

Sun H, Hashino E, Ding DL, Salvi R (2001) Reversible and irreversible damage to cochlear afferent neurons by kainic acid excitotoxicity. J Comp Neurol 430:172-181.

Takasaka T, Smith C (1971) The structure and innervation of the pigeon's basilar papilla. J Ultrastruct. Res 35:20-65.

Trautwein P, Hofstetter P, Wang J, Salvi R, Nostrant A (1996) Selective inner hair cell loss does not alter distortion product otoacoustic emissions. Hear Res 96:71-82.

Viana LM, O'Malley JT, Burgess BJ, Jones DD, Oliveira CACP, Santos F, Merchant SN, Liberman LD, Liberman MC (2015) Cochlear neuropathy in human presbycusis: confocal analysis of hidden hearing loss in postmortem tissue. Hear Res 327:78-88.

Wang J, Powers NL, Hofstetter P, Trautwein P, Ding D, Salvi R (1997) Effects of selective inner hair cell loss on auditory nerve fiber threshold, tuning and spontaneous and driven discharge rate. Hear Res 107:67-82.

Wong SJ, Abrams KS, Amburgey KN, Wang Y, Henry KS (2019) Effects of selective auditory-nerve damage on the behavioral audiogram and temporal integration in the budgerigar. Hear Res 374:24-34.

Wu PZ, Liberman LD, Bennett K, Gruttola VD, Malley JTO, Liberman MC (2019) Primary neural degeneration in the human cochlea: evidence for hidden hearing loss in the aging ear. Neuroscience 407:8-20.

Yeend I, Beach EF, Sharma M, Dillon H (2017) The effects of noise exposure and musical training on suprathreshold auditory processing and speech perception in noise. Hear Res 353:224-236. 